\title{
Very-Low-Frequency Radiation Spectra of Lightning Discharges
}

\author{
W. L. Taylor and A. G. Jean
}

(April 9, 1959)

\begin{abstract}
Spectral analyses are given of the groundwave portion of 33 sferic waveforms recorded from cloud-to-ground lightning discharges which occurred at distances ranging between about 150 and 600 kilometers from Boulder, Colo. Frequencies of peak energy lie between 5 and 20 kilocycles per second, which agree favorably with other published results. The average value of energy calculated from the groundwave pulses was found to be 26,600 joules, which is lower than values derived from other experiments. Various parameters, such as the peak amplitude and duration of the first half-cycle, are related to the radiated energy of the stroke.
\end{abstract}

\section{Introduction}

Appleton et al. (1926) were probably the first workers to utilize the cathode-ray oscilloscope in studying the electromagnetic energy emitted from lightning discharges. Following the development of radio communication techniques, it became evident that the lightning discharge was the source of interference to radio communication circuits.

In recent years, the radio signals emitted from lightning discharges, called atmospherics, or sferics, have been utilized as a source of signals in propagation studies at very-low radiofrequencies.

Various workers in England have been particularly active in the utilization of sferics in propagation studies, and it would be difficult and exhaustive to properly credit all of their accomplishments. However, as the work continued, it was learned that some atmospheric waveforms could be interpreted in terms of the reception of a series of pulses due to successive reflections between the earth and the ionosphere. It became evident that the character of the atmospheric waveform observed at great distances was materially altered by the propagation effects, while at shorter ranges, the waveform was more representative of the source function.

At the present time the use of atmospherics in propagation studies at the very-low radiofrequencies is common. NBS Boulder Laboratories have established a network of stations $[1]^{1}$ for this purpose, and the material presented in this paper resulted from the simultaneous observation of atmospheries at the Bureau recording sites.

The objective of this paper is to present data on Fourier spectra of the electric field strength of signals radiated from return-stroke lightning discharges. The radiation spectra of lightning discharges were determined from observations of the groundwave portion of the atmospheric. The precautions taken in identifying and utilizing groundwave pulses for this purpose are described in this paper.

1 Figures in brackets indicate the literature references at the end of this paper.

\section{Equipment and Collection of Data}

Stations at Boulder, Colo.; Salt Lake City, Utah; and Palo Alto, Calif.; were equipped to record the directions of arrival of atmospheric pulses, the pulse waveforms, and timing marks from which the times of arrival of individual pulses could be determined. Atmospheric waveforms used in the spectral analyses were recorded from vertical antennas. The overall amplitude response of the vertical antenna channel was constant within $\pm 1 \mathrm{db}$ over the bandpass and sloped to 3 -db cutoff points at frequencies of 1 and $100 \mathrm{kc}$. The phase response of this channel closely approximated a linear function of frequency within the bandpass.

A pair of vertical electrostatically shielded loop antennas, arranged at right angles to each other, were used in a direction-finding system at each station. The overall bandpass of each loop-antenna channel extended from 1 to $100 \mathrm{kc}$, and closely approximated that of the vertical-antenna channel. The direction-finding indications were obtained using the entire bandwidth of the loop-antenna channels. It was determined in earlier experiments [2] with wideband direction finders, that in many cases the groundwave component of the atmospheric could be discerned from the skywave components by virtue of differences in polarization and times of arrival of the groundwave and skywave pulses. $^{2}$ A time resolution of approximately 10 $\mu$ sec is afforded by the wideband direction-finder, which is adequate to resolve the groundwave and skywave pulses. The direction of arrival of the atmospheric, as indicated by the vertically polarized groundwave component, should be virtually free from error due to the presence of horizontally polarized components in the atmospheric.

Timing marks derived from a secondary frequency standard, synchronized with the standard timing emissions from station WWV, were recorded on the atmospheric waveform records. Using these timing

${ }^{2}$ Excellent discussions regarding the location of sferic sources through the use of radio direction-finding have appeared in the literature; Horner [3], in particular, has discussed the probable sources of error in direction-finding, including that resulting from the reception of horizontally polarized components of ionospheric waves. 
marks, the time of arrival of atmospheries could be determined at each station to an accuracy of about \pm 1 msec. Atmospheric waveforms resulting from a particular lightning discharge were located on the photographic records made at each station by virtue of their times of reception and directions of arrival.

The sweep of the oscilloscopes used in recording the atmospherics was activated by signals having amplitudes of about $50 \mathrm{mv} / \mathrm{m}$ or greater. Signals of smaller amplitude did not activate the sweep and therefore were not recorded. This feature provided some discrimination against atmospherics of relatively low amplitude; such as those of distant origin as well as some local cloud-to-cloud and precursory discharges. To minimize loss of early detail in the waveform, a $24-\mu$ sec delay line was used to delay recording the signal until after the sweep was initiated. The dynamic range of the recording equipment, in terms of the strength of a vertically polarized wave incident at the monopole antenna, extended from 0.2 to $6 \mathrm{v} / \mathrm{m}$. Most of the analyzed atmospherics had peak field amplitudes approaching $1 \mathrm{v} / \mathrm{m}$.

The locations of lightning discharges were determined by triangulation, using the direction of arrival indicated at each station. Due to the geometric arrangement of the 3-station network, the locations of lightning discharges which occurred near a recording station are relatively inaccurate; however, for distances greater than about $150 \mathrm{~km}$, the error is estimated to be equal or less than 10 percent of the range.

It was desired to identify waveforms on the photographic records that resulted from vertical lightning discharges. Two tests that were used in an attempt to achieve this selection are described below. The relative amplitude of the vertical component of the electric field which would be registered at the appropriate ranges from a vertical discharge was calculated using range information available from the directionfinding "fixes," and from groundwave propagation curves in the literature [15]. The source was considered to be vertically polarized if the relative values of the observed and calculated pulse amplitudes agreed within about 20 percent.

If a vertically polarized wave arrives at grazing incidence, the direction-finder response will approximate a straight line inclined at an angle proportional to the azimuthal direction of arrival of the wave. An incident wave consisting of both horizontal and vertical components of the electric field will cause an elliptical direction-finding pattern to be produced. The direction-finder responses to the atmospheric pulses which were selected for analyses indicated that the groundwave pulses were vertically polarized, and the first-hop skywave pulses were essentially, but not entirely, vertically polarized. Atmospheric waveforms which satisfied both the "amplitude" and "ellipticity" criteria outlined above were assumed to result from vertical lightning discharges.

No sferics were analyzed which originated at distances less than $150 \mathrm{~km}$ from Boulder, due, in part, to limitations in the accuracy of "fixes" at short ranges, and also to minimize contributions by the induction field at frequencies near $1 \mathrm{kc}$. It was desirable to limit the maximum observation range in order to minimize the distortion of the groundwave pulse by propagation and to reduce the interference caused in the groundwave by the first-hop skywave pulse.

The waveforms of two sferics, representative of those used in this analysis, are reproduced in figure 1a. The range to the source is approximately 165 $\mathrm{km}$ from both sferics 1 and 2 . The groundwave portion of sferic 1 is believed to comprise the first $110 \mu$ sec of the approximate $450-\mu$ sec time base. The earliest evidence of the arrival of the first-hop skywave pulse in sferic 1 occurs at $147 \mu$ sec. Note that the initial half cycle of the skywave pulse is negative, or opposite to the polarity of the first half-cycle of the groundwave pulse. This reversal in polarity of the initial part of the first-hop skywave pulses evident in figures $1 \mathrm{a}$ and $1 \mathrm{~b}$ is a consequence of the pseudo Brewster angle in ionospheric reflection. This phenomenon was discussed in an earlier paper [1].

The height of the ionospheric reflection was calculated to be about $65 \mathrm{~km}$, corresponding to a range of $165 \mathrm{~km}$ and a $147-\mu \mathrm{sec}$ time interval between the reception of the groundwave and the first-hop skywave pulses. In this calculation, the ionosphere was assumed to be a homogeneous ionized medium with a sharp lower boundary [4].

Note that sferic 2 is similar in character to sferic 1 , but the groundwave is of larger amplitude and longer duration. The first-hop skywave pulse was received before the entire groundwave pulse was recorded. It was necessary in such cases to complete the groundwave pulse as indicated by the dotted line before undertaking the spectral calculations. Since a very small fraction of the total area enclosed by the waveform is contained under the sketched-in portion, the error introduced into the spectral calculations is small.

Waveforms of sferics 17 and $18,{ }^{3}$ which were received at ranges of $460 \mathrm{~km}$ under nighttime conditions, are given in figure $1 \mathrm{~b}$. Note that in these

3 Numbers were assigned to the atmospherics in order of their selection for analyses and each number associates an atmospheric with its spectrum. Sferics $1,2,17$, and 18 were selected to represent the variability encountered in the pheries (sferics 3 through 16 and 19 through 33 ) are not presented in this report.

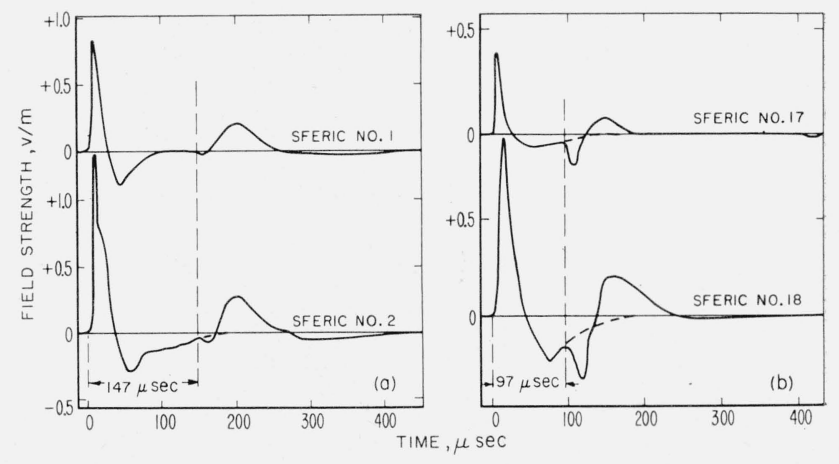

FIGURE 1. Representative atmospheric waveforms. 
cases the time interval between reception of the groundwave and the first-hop skywave pulses is about $97 \mu$ sec compared to an interval of $147 \mu \mathrm{sec}$ in figure 1a. As a consequence of the shorter time interval between the reception of the groundwave and first-hop skywave pulses, a larger portion of the groundwave pulses are concealed by the skywave pulses than for the sferics 1 and 2. Prior to performing the spectral analyses of the groundwave pulses for sferies 17 and 18, the trailing edges of the groundwave pulses were sketched in as indicated. A larger error resulted in such cases; e.g., the area under the sketched-in portion in sferic 18 is approximately 13 percent of the total area enclosed by the waveform of the groundwave pulse; however, this error is not considered to be serious in the present analyses.

The ionospheric reflection height for these nighttime observations was calculated to be about $80 \mathrm{~km}$, using a range of $460 \mathrm{~km}$ and a time interval between reception of groundwave and first-hop skywave pulses of $97 \mu \mathrm{sec}$.

\section{Data Analyses}

Let $G(t)$ represent the instantaneous value of the vertical component of the electric field in units of volts per meter. Also let the waveform start at time $t=0$, so that

$$
G(t)=0, \quad \text { for } t \leq 0,
$$

and the end of the pulse occurs at $t=\tau$ so that

$$
G(t)=0, \quad \text { for } t \geq \tau .
$$

The complex spectrum of the pulse can be represented by the Fourier in tegral

$$
F(f)=\int_{0}^{\tau} e^{-j 2 \pi f t} G(t) d t
$$

where $f$ represents the frequency in cycles per second and $t$ is the time in seconds after the beginning of the pulse.

The complex spectral components of the atmospheric were calculated at intervals of $1 \mathrm{kc}$ on an electronic computer. The absolute value, $|F(f)|$, of the spectra of the groundwave pulses of individual atmospherics are given in figures $2 \mathrm{a}$ through $2 \mathrm{~h}$. Fourteen of the analyzed pulses were received under daylight conditions from a storm approximately 165 $\mathrm{km}$ from Boulder, and 19 pulses were received under nighttime conditions from ranges varying between 400 and $620 \mathrm{~km}$ from Boulder. The approximate range at which each pulse was observed is indicated on the appropriate figure.

The frequencies of maximum energy for return stroke lightning discharges have been reported by a number of different workers. ${ }^{4}$ Their separate findings indicate that the frequency peaks occur in the 5- to 20 -kc region. It will be observed that the peak fre-

${ }^{4} \mathrm{It}$ is difficult to adequately reference all of the available literature dealing with this subject. A few representative references [5-12] have been selected.
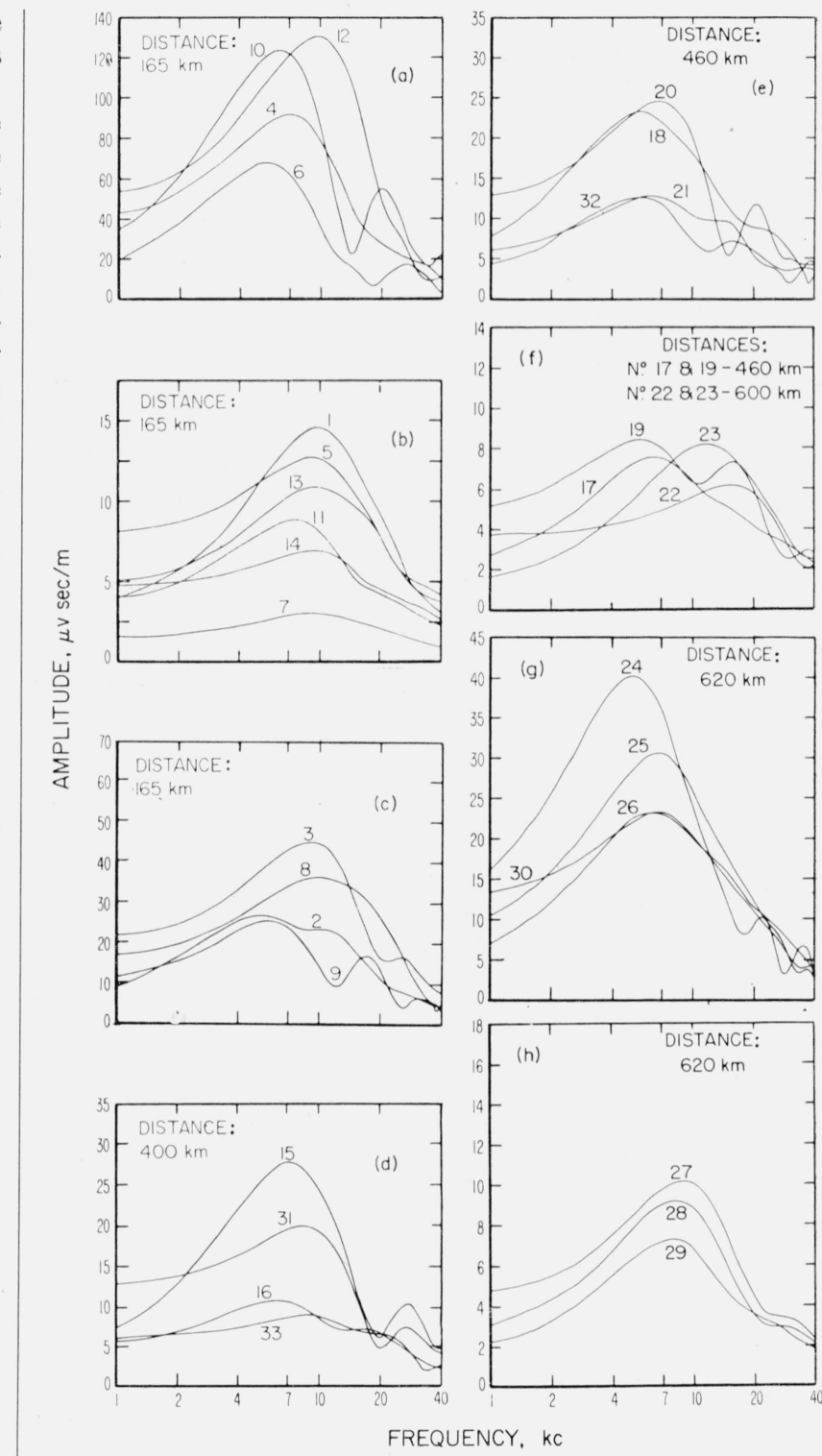

Figure 2. Amplitude spectra of atmospherics (see text).

quencies in figure 2 fall within this range of frequencies.

With a knowledge of the complex spectrum $F(f)$ of a groundwave pulse observed at distance $d_{1}$, and of the appropriate values of earth constants, it is possible to calculate the spectrum $|F(f)|$ of a groundwave pulse at a different range, $d_{2}$, using methods evolved by Wait [14]. This rigorous method was not followed in this paper, due to the complex nature of the calculations and the lack of adequate information concerning earth constants. The less rigorous method used in normalizing the spectra $|F(f)|$ to a common distance is described below.

The atmospheric pulses were recorded at distances between 150 and $600 \mathrm{~km}$ and their spectral peaks 
were attained at frequencies near $10 \mathrm{kc}$. A uniform earth, having a conductivity of about $10^{-2} \mathrm{mho} / \mathrm{m}$, was assumed to exist along the propagation paths. Referring to the curves of groundwave amplitude versus distance [15], it is evident for these values of earth conductivity, distance, and frequency, that the signal amplitude decreases approximately at the inverse distance rate. Accordingly, the individual spectra were referred to a common distance of $1 \mathrm{~km}$ by increasing each spectral component in proportion to the observation range. The spectrum shown in figure 3 is the average of the individual spectra normalized to this distance. The deviation of the individual spectra from the average is not very large which seems to support evidence in the literature $[12,13]$ that the radiation spectra of return-stroke lightning discharges do not vary a large amount from event to event. The $1-\mathrm{km}$ value was selected as a matter of convenience; the spectrum in figure 3 is not intended to represent the actual distribution of amplitude with frequency at that distance.

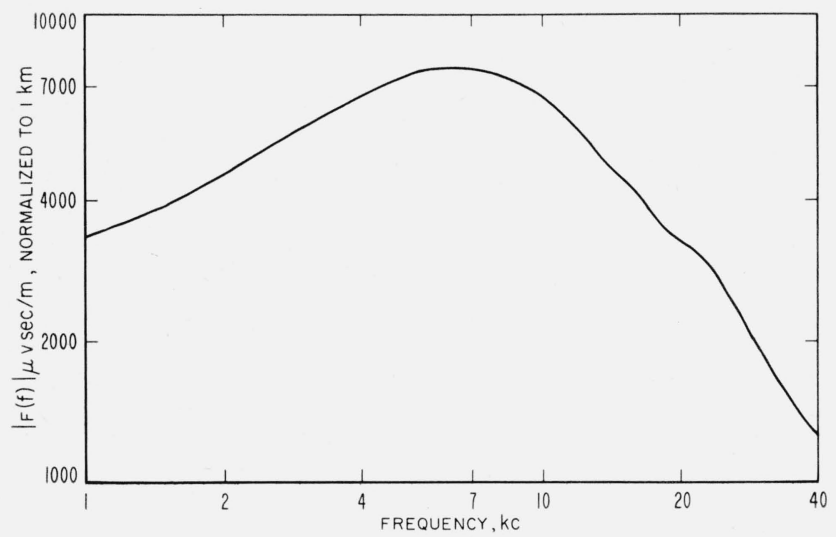

Figure 3. Average of amplitude spectra, normalized to $1 \mathrm{~km}$.

The peak amplitude of this spectrum occurs at a frequency of $6.5 \mathrm{kc}$; at frequencies below the peak, the spectral amplitude is proportional to the 0.4 power of the frequency, while at frequencies higher than the peak, the spectral amplitude decreases as the inverse 1.5 power of the frequency.

The total energy available in a lightning discharge has been estimated to be about $2 \times 10^{17}$ ergs [16]. This large amount of energy is partitioned into a number of different forms; for example, some energy is required to ionize the gas in the discharge channel, a larger portion is liberated as frictional heat in the movement of gaseous components, and some is evident as electromagnetic radiation, etc. An appreciable part of the energy is consumed in "ground losses" due to the flow of induced currents in the earth [17].

For the purposes at hand, it was desired to estimate the amount of vlf radio energy radiated into the earth's atmosphere (i.e., exclusive of earth losses) by the lightning discharges. This form of energy is a very small portion of the total amount available.
The energy density, $E_{d}$, of the transient $G(t)$ is represented in joules/meter ${ }^{2}$ by

$$
E_{d}=\frac{1}{120 \pi} \int_{0}^{\infty} G(t)^{2} d t
$$

It was assumed that the current at the source was a simple linear dipole oriented along the vertical axis. The radiation component of the dipole electric field is proportional to $\cos \theta$, where $\theta$ is the angle of elevation measured from the horizontal. The vlf energy, $E_{T}$, in joules, radiated into the atmosphere was obtained by integrating the energy density $E_{d}$ over the surface of the radiation pattern of the source. Thus,

$$
\begin{aligned}
E_{T} & =\int_{0}^{2 \pi} \int_{0}^{\pi / 2} E_{d} r^{2} \cos ^{3} \theta d \theta d \phi \\
& =4 / 3 \pi r^{2} E_{d},
\end{aligned}
$$

where $\theta$ is the angle of elevation measured from the horizontal, $\phi$ is the angle measured in the equatorial plane, and $r$ is the distance in meters from the source to the point of observation.

Values of energy represented by each of the pulses, calculated using expression (3), are given in table 1 .

TABLE 1. Very-low-frequency energy radiated into the atrosphere by return-stroke lightning discharges

\begin{tabular}{c|c|c|c}
\hline $\begin{array}{c}\text { Atmos- } \\
\text { pheric No. }\end{array}$ & $\begin{array}{c}\text { Dis- } \\
\text { tance }\end{array}$ & $f_{0}$ & $E_{T}$ \\
\hline
\end{tabular}

\begin{tabular}{|c|c|c|c|}
\hline & $\mathrm{km}$ & $k c$ & $j$ \\
\hline 1. & 165 & 10.0 & 2. $2 \times 10^{3}$ \\
\hline 2. & 165 & 5.0 & 6. $2 \times 10^{3}$ \\
\hline 3 & 165 & 9.0 & 1. $7 \times 10^{4}$ \\
\hline 4 & 165 & 7.0 & 5. $5 \times 10^{4}$ \\
\hline 5. & 165 & 9.0 & 1. $8 \times 10^{3}$ \\
\hline 6. & 165 & 5.5 & 2. $1 \times 10^{4}$ \\
\hline 7. & 165 & 9.5 & 1. $1 \times 10^{2}$ \\
\hline 8 & 165 & 10.0 & 1. $5 \times 10^{4}$ \\
\hline 9 & 165 & 6.0 & 4. $3 \times 10^{3}$ \\
\hline 10 & 165 & 6.0 & $8.8 \times 10^{4}$ \\
\hline 11 & 165 & 7.5 & $6.9 \times 10^{2}$ \\
\hline 12 & 165 & 9.5 & 1. $3 \times 10^{5}$ \\
\hline 13 & 165 & 10.0 & 1. $5 \times 10^{3}$ \\
\hline 14 & 165 & 9.5 & 5. $9 \times 10^{2}$ \\
\hline
\end{tabular}

Sferic groundwave recorded at Boulder duriıg daylight July 7,1959

\begin{tabular}{|c|c|c|c|}
\hline 15 & 400 & 7.5 & $2.1 \times 10^{4}$ \\
\hline 16 & 400 & 6.0 & 6. $5 \times 10^{3}$ \\
\hline 31 & 400 & 8.5 & $2.0 \times 10^{4}$ \\
\hline 33 & 400 & 8.5 & 5. $5 \times 10^{3}$ \\
\hline 17 & 460 & 6.5 & 4. $5 \times 10^{3}$ \\
\hline 18. & 460 & 5.5 & $2.9 \times 10^{4}$ \\
\hline 19 & 460 & 5.5 & 6. $2 \times 10^{3}$ \\
\hline 20 & 460 & 7.0 & 3. $1 \times 10^{4}$ \\
\hline 21 . & 460 & 7.0 & $1.1 \times 10^{4}$ \\
\hline 32 & 460 & 5. 5 & $9.3 \times 10^{3}$ \\
\hline 22 & 600 & 15.5 & $7.9 \times 10^{3}$ \\
\hline 23 & 600 & 12.0 & $1.1 \times 10^{4}$ \\
\hline 24 & 620 & 5.5 & $1.1 \times 10^{5}$ \\
\hline 25 & 620 & 7.0 & 9. $2 \times 10^{4}$ \\
\hline 26 & 620 & 6.5 & $6.4 \times 10^{4}$ \\
\hline 27 & 620 & 9.0 & 1. $3 \times 10^{4}$ \\
\hline 28 & 620 & 8.5 & 9. $6 \times 10^{3}$ \\
\hline 29 & 620 & 8.0 & $6.4 \times 10^{3}$ \\
\hline $30 \ldots$ & 620 & 6.5 & 6. $3 \times 10^{4}$ \\
\hline
\end{tabular}

Sferic groundwave recorded at Boulder during nighttime July 26,1958 
The average of these values of energy is $26,600 \mathrm{j}$. On another occasion [10], sferics were received from thunderstorms in a frontal region extending through Texas, Louisiana, and Oklahoma. The average of the total radiated energy values calculated from seven of these sferics was about $250,000 \mathrm{j}$; the individual values range from 130,000 to $500,000 \mathrm{j}$. Laby, et al. [11], reported the average value of energy radiated from 35 sferics observed in Australia was 200,000 j. It appears that the sferics used in the present work resulted from less energetic discharges than those reported by Taylor and by Laby. It is not at all certain that the energy value reported here is representative of return-stroke discharges from thunderstorms occurring in the Rocky Mountain area. However, part of the differences between the energy values quoted might have resulted from differences in thunderstorm characteristics, differences in topography, earth conductivity, etc.

The importance of land elevation in determining thunderstorm characteristics was pointed out by Loeb [18]. In general, the lightning discharges used in this analysis occurred over land of higher elevation than those previously reported by Taylor. It is not known in what area of Australia the lightning flashes occurred that were used in Laby's analysis.

It is interesting to note that the ratio of the total energy available in a lighting discharge to the values of electromagnetic energy radiated into the atmosphere is as large as $10^{5}$ or $10^{6}$.

A wide variation was noted in the detail of the individual groundwave pulses; however, most of them consisted of two half-cycles. The first half-cycles were larger in amplitude and shorter in duration than the corresponding values in the second halfcycle. These two characteristics of the first halfcycle would be expected to control the total radiated energy to a certain extent. After normalizing the observations to a $1-\mathrm{km}$ range, the relationships existing between various parameters mentioned above were examined. Some of these comparisons are described below.

(A) Frequency of spectral peak versus total radiated energy. The relationship between the frequency of spectral peak $\left(f_{0}\right)$ and the total radiated energy $\left(E_{T}\right)$ is given in figure 4. Although the data points scatter, there is a slight tendency for the more energetic sferics to attain energy peaks at the lower frequencies.

(B) Peak amplitude of sferics versus total radiated energy. The peak amplitude of the groundwave pulse $\left(E_{\max }\right)$ is plotted as a function of total radiated energy in figure 5. There is a close relationship between these parameters. The straight line drawn through the data points relate total radiated energy to the 2.4 power of the peak field strength.

(C) Spectral peak versus total radiated energy. The relationship between the peak of the spectrum of the groundwave pulse, $F(f)_{\max }$, versus the total radiated energy of the pulse is given in figure 6 . A close relationship is indicated between these parameters. The straight line through the data points indicates that the energy is proportional to the 1.8 power of

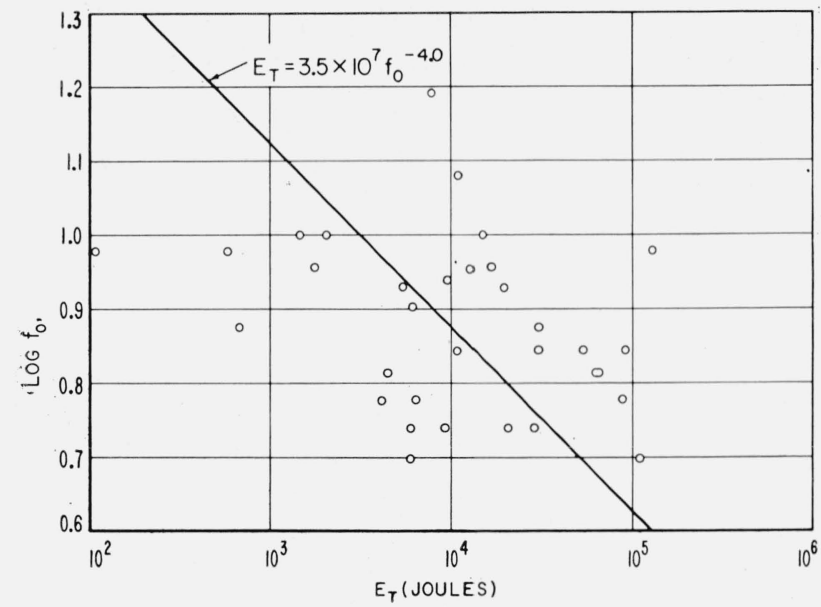

FIGURe 4. Frequency of spectral peak versus total radiated energy.

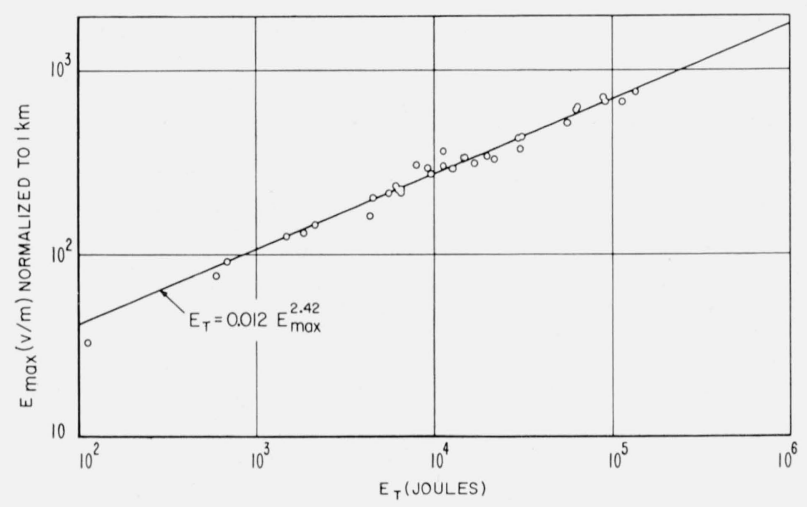

Figure 5. Pulse amplitude versus total radiated energy.

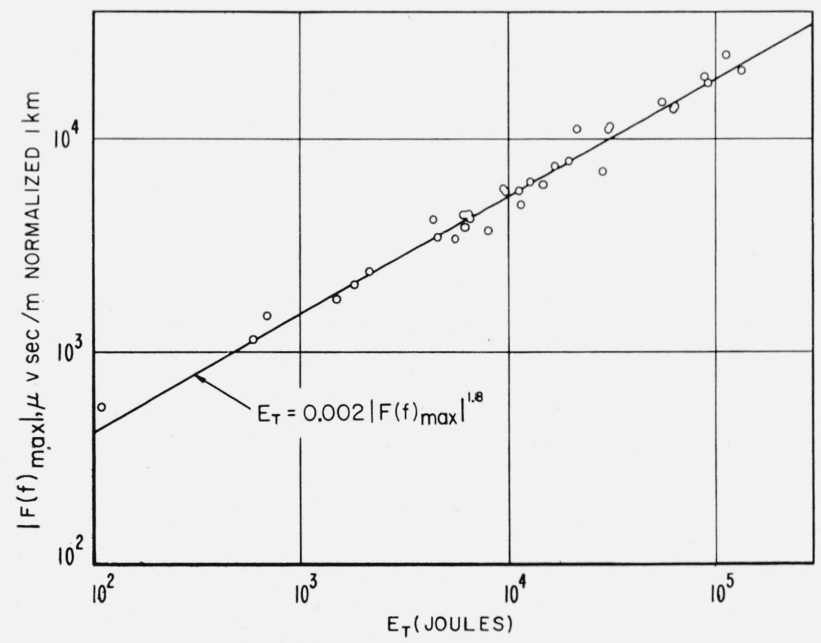

FiguRe 6. Spectral peak versus total radiated energy.

the spectral peak. The frequency of the spectral peak is not explicitly accounted for in this relationship.

(D) Length of first half-cycle versus total radiated energy. In figure 7 the relationship between the length of the first half-cycle of the groundwave pulse 


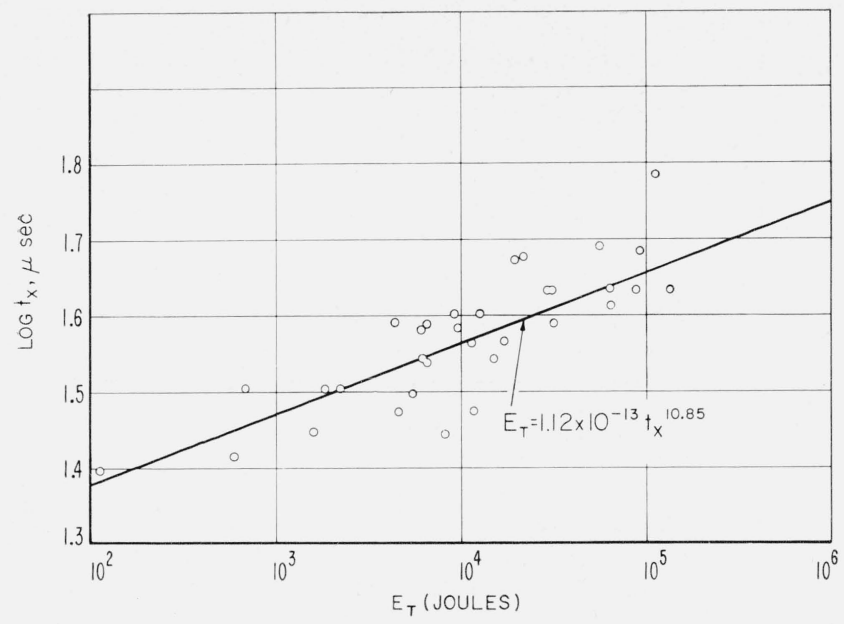

Figure 7. Length of first half-cycle versus total radiated energy.

( $t_{x}$ in microseconds) and the corresponding value of total radiated energy is plotted. The relative importance of the first half-cycle in determining the energy is indicated in this plot and in figure 5, in which the peak amplitude of the groundwave pulse is plotted as a function of total radiated energy.

The field strength of a pulse is related to the rate of change of the source moment [13, 14, 19], hence, flashes having rapidly rising currents would be expected to produce sferics of high peak amplitude. The duration of the first half-cycle of the groundwave pulse should correspond to the time at which the source current reached its maximum value. Hence, sferics of large energy result from rapidly rising and long continuing source currents. It would be expected that the more energetic discharges would produce sferies of longer duration, and a strong tendency of this nature is observed in the data; however, it was also observed that sferics representing relatively low values of energy attain spectral peaks within the same frequency region, 5-20 kc, as those of larger energy.

The close relationship existing in figure 5 between the peak amplitude of the first half-cycle and total radiated energy suggests the possibility of gaining a useful estimate of energy by observing the peak amplitude of the groundwave pulse and by determining the distance to the flash. However, it is not known that this relationship will hold equally well at the higher energy levels.

It would be of interest to compare the characteristics of sferics originating (1) over land of different elevations and those originating over sea water, and (2) from frontal and air-mass thunderstorms. This would aid in appraising the influence of topography, earth conductivity, and meteorological factors in determining the characteristics of sferics.

Atmospheric waveforms recorded at Boulder were used in the present analysis. Simultaneous recordings of these atmospherics are also available from the other stations located at Stanford and Salt Lake City. Analyses of this data is continuing to de- termine values of propagation attenuation [10] and phase velocity of propagation $[20,21]$.

The authors wish to acknowledge the mathematical calculations made by L. Jerome Lange and A. Murphy, the preparation of the programing for the computer by H. H. Howe, and helpful conversations with Roger Gallet and James R. Wait during the preparation of this paper.

\section{References}

[1] A. G. Jean, L. J. Lange, and J. R. Wait, Ionospheric reflection coefficients at vlf from sferic measurements, Geofisica Pura e Applicata 38, 147 (1957).

[2] R. A. Helliwell, A. G. Jean, and W. L. Taylor, Some properties of lightning impulses which produce whistlers, Proc. IRE 46, 1760 (1958).

[3] F. Horner, The accuracy of the location of sources of atmospheries by radio direction finding, Proc. Inst. Elec. Engrs. (London) 101, Pt. III, 383 (1954).

[4] J. R. Wait and A. Murphy, Multiple reflections between the earth and the ionosphere in vlf propagation, Geofisica Pura e Applicata 35, 61 (1956).

[5] C. E. R. Bruce and R. H. Golde, The lightning discharge, J. Inst. Elec. Engrs. (London) 88, Pt. II, 487 (1941).

[6] H. Norinder, Variations of the electric field in the vicinity of lightning discharges, Arkiv Geofysik 1, 543 (1953).

[7] P. W. A. Bowe, The waveforms of atmospherics and the propagation of very low frequency radio waves, Phil. Mag. [7] 42, 121 (1951).

[8] F. W. Chapman and A. G. Edwards, Some observations on the frequency spectrum of atmospheric disturbances due to thundercloud discharges and their effect on resonant circuits, Proc. Union Radio Scientifique Internationale Comm. IV, No. 252 (1950) Zurich.

[9] A. Kimpara, The typhoon kezia and atmospherics, Proc. Japan Acad. 27, 366 (1951)

[10] W. L. Taylor and L. J. Lange, Some characteristics of vlf propagation using atmospheric waveforms, Second Conf. Atmospheric Elec. May 1958 (to be published April 1959).

[11] T. H. Laby, J. J. McNeill, F. G. Nicholls, and A. F. B. Nickson, Waveform energy and reflexion by the ionosphere of atmospherics, Proc. Roy. Soc. (London) [A] 174, 145 (1940).

[12] F. Chapman and R. C. V. Macario, Propagation of audiofrequency radio waves to great distances, Nature 17\%, 930 (1956).

[13] A. D. Watt and E. L. Maxwell, Characteristics of atmospheric noise from 1 to $100 \mathrm{ke}$, Proc. IRE 45, 787 (1957).

[14] J. R. Wait, On the waveform of a radio atmospheric at short ranges, Proc. IRE 44, 1052 (1956).

[15] J. R. Wait and H. H. Howe, Amplitude and phase curves for ground-wave propagation in the band 200 cycles per second to 500 kilocycles, NBS Circ. 574 (May 1956).

[16] C. W. Allen, Astrophysical quantities, p. 123 (Úniv. of London, Athlone Press, 1955).

[17] J. R. Wait, personal communication.

[18] L. B. Loeb, Thunderstorms and lightning strokes, p. 330 Modern physies for the engineer by Royal Weller and others (McGraw-Hill Book Co., New York, 1954).

[19] H. A. Thomas and R. E. Burgess, Survey of existing information and data on radio noise over the frequency range 1-30 Mc/s, DSIR Radio Research, Special Rept. 15, p. 38 (1947).

[20] J. R. Wait, Propagation of very-low-frequency pulses to great distances, J. Research NBS 61, 187 (1958) RP2898.

[21] Ia. L. Al'Pert and S. V. Borodina, Zhur. Eksptl. i Teoret. Fiz. (J. Exp. Theoret. Phys. U.S.S.R.) 33, 1305 (1957).

Boulder, Colo.

(Paper 63D2-18). 